\title{
Constructing Approximate Shortest Path Maps in Three Dimensions*
}

\author{
Sariel Har-Peled ${ }^{\dagger}$
}

\section{Abotract}

We present a new technique for constructing a data-structure that approximates shortest path maps in $\mathbb{R}^{d}$. By applying this technique, we get the folloving two results on approximate shorte日t path maps in $\mathbb{R}^{3}$.

(i) Given a polyhedral surface or a convex polytope $\mathcal{P}$ with $n$ edges in $\mathbb{R}^{3}$, a source point $s$ on $\mathcal{P}$, and a real parameter $0<\varepsilon \leq 1$, we present an algorithm that computes a subdivision of $\mathcal{P}$ of size $O((n / \varepsilon) \log (1 / \varepsilon))$ which can be used to answer efficiently approximate shortest path querico. Namely, given any point $t$ on $\mathcal{P}$, one can compute, in $O(\log (n / \varepsilon))$ time, a distance $\Delta p_{1} a(t)$, such that $d_{\mathcal{P}_{1} s}(t) \leq \Delta_{\mathcal{P}_{1}}(t) \leq(1+\varepsilon) d_{\mathcal{P}_{, s}}(t)$, where $d_{\mathcal{P}_{, \theta}}(t)$ is the length of a shortest path between $s$ and $t$ on $\mathcal{P}$.

The map can be computed in $O\left(n^{2} \log n+(n / \varepsilon) \log (1 / \varepsilon)\right.$ $\log (n / e))$ time, for the case of a polyhedral surface, and in $O\left(\left(n / e^{3}\right) \log (1 / \varepsilon)+\left(n / e^{1.5}\right) \log (1 / \varepsilon) \log n\right)$ time if $\mathcal{P}$ is a convex polytope.

(ii) Given a net of polyhedral obstacles $\mathcal{O}$ with a total of $n$ edges in $\mathbb{R}^{3}$, a source point $s$ in $\mathbb{R}^{3} \backslash$ int $\bigcup_{o \in \mathcal{O}} O$, and a rcal parameter $0<\varepsilon \leq 1$, we present an algorithm that computen a subdivision of $\mathbb{R}^{3}$, which can be used to answer efficiently approximate shortest path queries. That is, for any point $t \in \mathbb{R}^{3}$, one can compute, in $O(\log (n / \varepsilon))$ time, a diatance $\Delta_{O_{1}}(t)$ that $\varepsilon$-approximates the length of a shorteat path from $a$ to $t$ that avoids the interiors of the obstacles. This subdivision can be computed in roughly $O\left(n^{4} / \varepsilon^{6}\right)$ time.

\section{Introduction}

The three-dimensional Euclidean shortest-path problem is defined as followa: Given a set of pairwise-disjoint polyhedral objects in $\mathbb{R}^{s}$ and two points $s$ and $t$, compute the shortest

Thin work has been supported by a grant from the U.S.Inrneli Binational Science Foundation. This work is part of the author's $\mathrm{Ph}$.D. thesis, prepared at Tel-Aviv University under the oupervision of Prof. Micha Sharir.

tgchool of Mathemetical Sciences, Tel Aviv University, Tel Aviv 60978, Inrael; dariol Omath. tan. ac. il

Permission to make digital or hard copies of all or part of this work for personal or classroom use is granted without fee provided that copies are not mado or distributed for profit or commercial advantage and that copics bear this notice and the full citation on the first page. To copy otherwise, to republish, to post on servers or to redistribute to lists, requirez prior cpecific permission and/or a fee.

sCO 98 Minneapolis Minnesota USA

Copyright ACM 1998 0.89791-973-4/98/6...\$5.00 path between $s$ and $t$ which avoids the interiors of the given polyhedral 'obstacles'. This problem has received considerable attention in computational geometry. It was shown to be NP-hard by Canny and Reif [3], and the fastest available algorithms for this problem run in time that is exponential in the total number of obstacle vertices (which we denote by $n$ ) $[20,21]$. The apparent intractability of the problem has motivated researchers to develop polynomial-time algorithms for computing approximate shortest paths and for computing shortest paths in special cases.

In the approximate three-dimensional Euclidean shortestpath problem, we are given an additional parameter $\varepsilon>0$, and the goal is to compute a path between $s$ and $t$ that avoids the interiors of the obstacles and whose length is at most $(1+\varepsilon)$ times the length of the shortest obstacleavoiding path (we call such a path an $\varepsilon$-approximate path). Approximation algorithms for the three-dimensional shortest path problem were first studied by Papadimitriou [19], who gave an $O\left(n^{4}(L+\log (n / \varepsilon))^{2} / \varepsilon^{2}\right)$-time algorithm for computing an $\varepsilon$-approximate shortest path, where $L$ is the number of bits used in each computation. A rigorous analysis of Papadimitriou's algorithm was recently given by Choi et al. [6]. A different approach was taken by Clarkson [7] resulting in an algorithm with roughly $O\left(n^{2} / \varepsilon^{4}\right)$ running time (the precise result is stated in Theorem 4.1).

The problem of computing a shortest path between two points along the surface of a single convex polytope is an interesting special case of the three-dimensional Euclidean shortest-path problem. Sharir and Schorr [22] gave an $O\left(n^{3} \log n\right)$ algorithm for this problem, exploiting the property that a shortest path on a polyhedron unfolds into a straight line. Mitchell et al. [16] improved the running time to $O\left(n^{2} \log n\right)$; their algorithm works for non-convex polyhedra (or polyhedral surfaces) as well. Chen and Han [5] gave another algorithm with an improved running time of $O\left(n^{2}\right)$. It is a rather long-standing and intriguing open problem whether the shortest path on a convex polytope can be computed in subquadratic time. This has motivated the problem of finding near-linear algorithms that produce only an approximation of the shortest path. The first result in this direction is by Hershberger and Suri [12]. They present a simple algorithm that runs in $O(n)$ time, and computes a path whose length is at most $2 d_{P}(s, t)$. Using the algorithm of [12], Agarwal et al. [1] present a relatively simple algorithm that computes an $E$-approximate shortest path (i.e., a path on $\partial P$ between two points $s, t \in \partial P$ whose length is at most $\left.(1+\varepsilon) d_{P}(s, t)\right)$, for any prescribed $0<\varepsilon \leq 1$, where the running time of the algorithm is $O\left(n \log (1 / \varepsilon)+1 / \varepsilon^{3}\right)$. In a companion paper [11], we present an improved algorithm, 
with $O(n)$ preprocessing time, that answers two-points $\varepsilon$ approximate shortest-path queries in $O\left((\log n) / \varepsilon^{1.5}+1 / \varepsilon^{3}\right)$ time, for any pair of points $s, t \in \partial P$. Recently, Varadarajan and Agarwal [24] gave a subquadratic time algorithm that computes a constant approximation to the shortest path on a polyhedral terrain. Other recent works, by Mata and Mitchell [14], and also by Lanthier at al. [13] implement various heuristics for computing approximate shortest paths on weighted terrains (i.e., each face $f$ is being assigned a weight $w_{f}$, such that the distance between any two points $a, b \in f$ is $\left.w_{f} \cdot|a b|\right)$. Those programs give satisfactory results in prectice, which are within an order of magnitude better than their worst case analysis.

In this paper, estending our work in [11], we present a new general technique for constructing a data-structure that one can use to answer $\varepsilon$-approsimate shortest-path queries, for a source point $s$ and approximation factor $\varepsilon>0$ fixed in advance. Using this technique, we solve two problems involving approximate shortest path maps in $\mathbb{R}^{3}$.

Approximate shortest path maps. The exact algorithms of $[16,22]$ receive as input a convex polytope or a polyhedral surface $\mathcal{P}$, and a fixed source point $s$ on $\mathcal{P}$, and compute a map (i.e., a subdivision of $\mathcal{P}$ ) of complexity $\Theta\left(n^{2}\right)$, that can be used to answer (exact) shortest path queries from 3 to any point on $\mathcal{P}$ (along $\mathcal{P}$ ) in $O(\log n$ ) time (such a query reports the length of the shortest path; reporting the path it self might require more time). This shortest path map can be stored in linear space, for the case of a convex polytope, by using a persistent data-structure, see [17]. However, the time required to compute this compact representation of the shortest path map is quadratic in the worst case. This raises the problem of computing a map of near-linear size for approsimate shortest-path queries from $\mathbf{s}$. We show in Section 3 that this is indeed possible: Given a polyhedral surfece $\mathcal{P}$ with $n$ edges in $\mathbb{R}^{3}$, a source point $s \in \mathcal{P}$, and a prescribed $0<c \leq 1$, there exists a map (a subdivision of $\mathcal{P}$ ) of complexity $O((n / s) \log (1 / \varepsilon))$, such that for any $t \in \mathcal{P}$, one can compute the length of an $\varepsilon$-approximate shortest path between $s$ and $t$ on $\mathcal{P}$ in $O(\log (n / \varepsilon))$ time, by locating $t$ in the map.

We present an algorithm that constructs such an approximation map in $O\left(n^{2} \log n+(n / \varepsilon) \log (1 / \varepsilon) \log (n / c)\right)$ time, for the case of a polyhedral surface, and in $O\left(\left(n / \varepsilon^{b}\right)\right.$ $\left.\log (1 / \varepsilon)+\left(n / \varepsilon^{1.5}\right) \log (1 / \varepsilon) \log n\right)$ time, for the case of a conves polytope. Note that if $\mathcal{P}$ is a convex polytope than our previous result [11] provides an alternative structure with similar properties. However, the dependence of the query time on $\varepsilon$ is much better in the method we present here.

Approximate spatial shortest path maps. In Section 4, we present a similar result for $\varepsilon$-approximate shortest paths among polyhedral obstacles in $\mathbb{R}^{3}$. Let $\mathcal{O}$ be a set of polyhedral obstacles in $\mathbb{R}^{3}$ with a total of $n$ edges, $s$ a source point in $\mathbb{R}^{3}$, and $0<\varepsilon \leq 1$ a parameter. We show that there exists a spatial subdivision $\mathcal{M}$ of $\mathbb{R}^{3}$, such that for any $t \in \mathbb{R}^{3}$, one can compute, in $O(\log (n / \varepsilon))$ time, the length of an $\varepsilon$-approximate shortest path between $s$ and $t$, that avoids the interiors of the obstacles, by performing a spatial point-location query with $t$ in $\mathcal{M}$. The space needed to compute and preprocess $\mathcal{M}$ for spatial point-location is $O\left(n^{2} / c^{4+\delta}\right)$, for any $\delta>0$, and the preprocessing time is

$$
O\left(\frac{n^{4}}{\varepsilon^{2}}\left(\frac{\beta(n)}{\varepsilon^{\varepsilon}} \log \frac{n}{\varepsilon}+\log (n \rho) \log (n \log \rho)\right) \log \frac{1}{\varepsilon}\right),
$$

where $\rho$ is the ratio of the length of the longest edge in $\mathcal{O}$ to the Euclidean distance between $s$ and $t, \beta(n)=$ $\alpha(n)^{O(\alpha(n))^{O(1)}}$, and $\alpha(n)$ is the extremely slowly growing inverse of the Ackermann function. This algorithm uves the algorithm of Clarkson [7], that computes an $c$-approximate shortest path, between two given points, in

$$
O\left(\frac{n^{2}}{\varepsilon^{4}} \beta(n) \log \frac{n}{\varepsilon}+n^{2} \log (n \rho) \log (n \log \rho)\right)
$$

time.

The paper is organized as follows. Section 2 introduces the notion of a distance function, and show how to compute a "small-size" additive weighted Voronoi diagram that enables us to $\varepsilon$-approximate this function. We present two applications of this result. In Section 3, we present the algorithm mentioned above for constructing a map for approximate shortest-path queries from a fixed source on a convex polytope or a polyhedral surface in $\mathbb{R}^{3}$. In Section 4 we present the algorithm mentioned above for constructing a spatial subdivision for approximate chortest-path querica from a fixed source among polyhedral obstacles in $\mathbb{R}^{3}$. We conclude in Section 5 by mentioning a few open problems.

\section{Approximating a Distance Function by a Weighted Voronoi Diagram}

In this section, we introduce the notion of a distance function, and show how to compute a "small-size" additive weighted Voronoi diagram that approximates it up to a factor of $1+\varepsilon$. We use this result in Sections 3 and Section 4 to derive our two main results.

Definition Let $\mathcal{I}$ be a subset of $\mathbb{R}^{d}$. A function $f: \mathcal{I} \rightarrow \mathbb{R}$ is a distance function on $\mathcal{I}$ if:

- $f(x)+f(y) \geq|x y|$, for any $x, y \in I$.

- $f(x)+|x y| \geq f(y)$, for any straight segment $x y \subseteq I$,

where $|x y|$ denotes the Euclidean distance between $x$ and $y$.

Thus, a distance function has to satisfy two typen of triangle inequalities. Since these inequalities are satiofied by the Euclidean distance from any fixed point, a distunce function can be regarded as a certain generalization of the Euclidean distance.

Example 2.1 Figure 1 illustrates some geometric restriction imposed on a univariate distance function.

Example 2.2 (i) Let $\mathcal{P}$ be a polyhedral surface in $\mathrm{IR}^{3}$, and let $s$ be a source point on $\mathcal{P}$. For any $t \in \mathcal{P}$, let $d_{\mathcal{P}, a}(t)$ denote the length of $a$ shortest path between $s$ and $t$ on $\mathcal{P}$. It is easy to verify that $d_{\mathcal{P}, a}(t)$ is a distance function on $\mathcal{P}$.

(ii) Let $\mathcal{O}$ be a collection of pairwise-disjoint polyhedral obstacles in $\mathbb{R}^{3}$, and let $s$ be a point in $F P(\mathcal{O})=\mathbb{R}^{3}$ । $\bigcup_{O \in \mathcal{O}}$ int $O$. Let $F P(\mathcal{O}, s)$ denote the connected component of $F P(\mathcal{O})$ that contains s (i.e., the set of all the points in $\mathbb{R}^{3}$ that can be connected to $s$ by a path that avoids the interiors of the obstacles of $\mathcal{O}$ ).

For any $t \in F P(\mathcal{O}, s)$, we denote by $d_{O, s}(t)$ the length of $a$ shortest path between $s$ and $t$, that avoids the interior of the obstacles of $\mathcal{O}$. Clearly, $d_{\mathcal{O}, a}$ is a distance function on $F P(\mathcal{O}, s)$. 


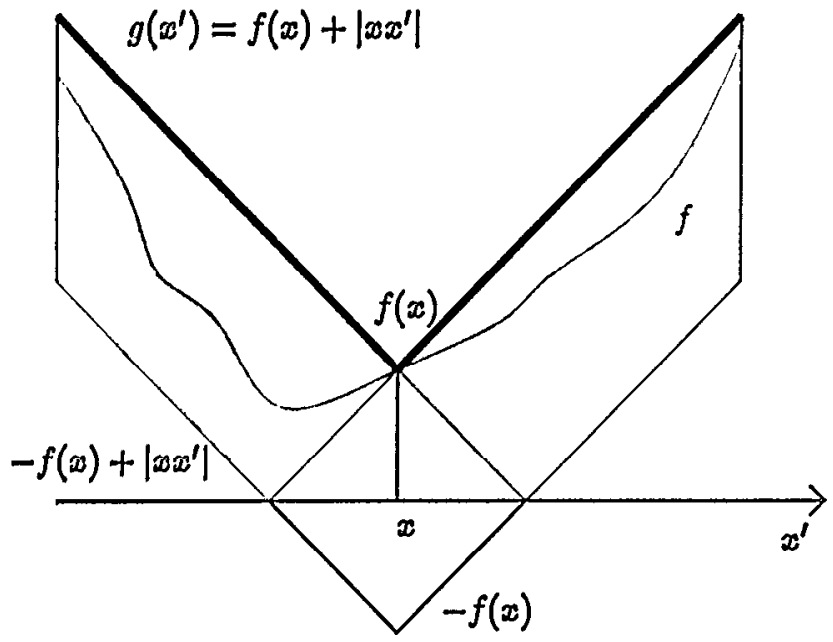

Figure 1: The graph of the distance function $f$ must lie invide the gray area. This implies that the function $g\left(x^{\prime}\right)=$ $f(x)+\left|x x^{\prime}\right|$ approximates $f\left(x^{\prime}\right)$ "well" in a small neighborhood of $x$, and for sufficiently large values of $x^{\prime}$.

Definition A pair $S=(S, w)$ is a weighted set of points if $S=\left\{p_{1}, \ldots, p_{m}\right\}$ is a finite set of points in $\mathbb{R}^{d}$, and $w(\cdot)$ in a function assigning non-negative weights to the points of $S$. We define the distance of a point $p$ from the point $p_{i}$ to be $V_{\left(p_{i} w\left(p_{i}\right)\right)}(p)=\left|p p_{i}\right|+w\left(p_{i}\right)$. We define $V_{S}(p)=$ $\min n_{i=1}^{m} V_{\left(p_{i}, w\left(p_{i}\right)\right)}(p)$. The function $V s(p)$ induces a natural aubdivision $\mathcal{V}_{\varepsilon}$ of $\mathbb{R}^{d}$ into cells, known as the (additive) weighted Voronoi diagram of $\mathcal{S}$, such that the $i$-th cell is the locun of all points closest to $p_{i}$ in this distance function. As is well lenown, in the planar case, $\mathcal{V}_{s}$ has complexity $O(m)$, and it can be computed in $O(m \log m)$ time (see [10]).

Remark 2.3 For $d \geq 3$, the complexity of an additive weighted Voronoi diagram of $m$ points in $\mathbb{R}^{d}$ is $O\left(m^{\lfloor d / 2\rfloor+1}\right)$. This follows by reducing the computation of the diagram to the computation of a convex hull in $d+2$ dimennions. Furthermore, one can compute the diagram in $O\left(m^{\lfloor d / 3\rfloor+1}\right)$ time. See [2].

We next show how to approximate a distance function $f(\cdot)$ by a weighted Voronoi diagram. First, we compute a global minimum $p_{0}$ of $f$. As illustrated in Figure 1 , the func-

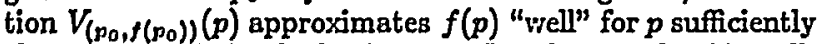
close to, or sufficiently far from $p_{0}$. In other vords, $f$ is wellapproximated in these regions by the weighted Voronoi diagram of the single site $p_{0}$ with weight $f\left(p_{0}\right)$. By adding extra aites to the diagram, we can make the distance induced by the reoulting diagram an $e$-approximation to $f$ everywhere, ab will be ohown next.

Definition Given a point $p \in \mathbb{R}^{d}$, and $r \geq 0$, let $B(p, r)$ denote the closed ball of radius $r$ centered at $p$, and let $\bar{B}(p, r)$ denote the set $\mathbb{R}^{d} \backslash B(p, r)$. For $r^{\prime}>r$, let $\mathcal{A}\left(p, r, r^{\prime}\right)$ denote the annulus (or shell) $B\left(p, r^{\prime}\right) \backslash B(p, r)$.

The following sequence of technical lemmas provide the basis for approximating a given distance function by a weighted Voronoi diagram. The following lemmas are stated for arbitrary dimension $d$. We vill apply them with $d=1,2$, or 3.
Lemma 2.4 Let $\mathcal{I}$ be a convex subset of $\mathbb{R}^{d}, f$ a distance function defined over $\mathcal{I}$, and $\mathcal{S}=(S, w)$ a weighted set, such that $S \subseteq I$ and $f(x) \leq v(x)$, for all $x \in S$. Then $f(t) \leq$ $V_{S}(t)$, for all $t \in \mathcal{I}$.

Proof: Let $t$ be any point of $I$, and let $x$ denote the point of $S$ realizing $V_{S}(t)$. Then $V_{S}(t)=w(x)+|t x| \geq$ $f(x)+|t x| \geq f(t)$.

Formalizing the intuition behind Figure 1, we have the following:

Lemma 2.5 Let $I$ be a convex subset of $\mathbb{R}^{d}, f$ a distance function defined over $\mathcal{I}, 0<\varepsilon \leq 1$ a parameter, $p$ a point in $\mathcal{I}$, and $w$ a real number such that $f(p) \leq w \leq(1+e / 8) f(p)$. Then $f(t) \leq V_{(p, w)}(t) \leq(1+\varepsilon) f(t)$, for all $t$ in

$$
\mathcal{I} \cap\left(B\left(p, \frac{\varepsilon f(p)}{8}\right) \cup \bar{B}\left(p, \frac{6 f(p)}{\varepsilon}\right)\right) .
$$

Proof: The first inequality $f(t) \leq V_{(p, w)}(t)$ follows immediately from Lemma 2.4 .

As for the other inequality, for $t \in \mathcal{I} \cap B\left(p, \frac{s f(p)}{B}\right)$, we have

$$
\begin{aligned}
\frac{w}{(1+\varepsilon / 8)}-\frac{\varepsilon w}{8} & \leq f(p)-|p t| \leq f(t) \leq V_{(p, w)}(t) \\
& =w+|p t| \leq w+\frac{\varepsilon w}{8} .
\end{aligned}
$$

However,

$$
\begin{aligned}
\frac{w+\varepsilon w / 8}{\frac{w}{1+c / 8}-\varepsilon w / 8} & =\frac{1+\varepsilon / 8}{\frac{1}{1+\varepsilon / 8}-\varepsilon / 8} \leq \frac{1+\varepsilon / 8}{1-\varepsilon / 8-\varepsilon / 8} \\
& =\frac{1+\varepsilon / 8}{1-\varepsilon / 4} \leq 1+\varepsilon,
\end{aligned}
$$

since $\varepsilon \leq 1$. Thus $V_{(p, w)}(t) \leq(1+\varepsilon) f(t)$, for all $t \in I \cap$ $B(p, \varepsilon f(p) / 8)$.

For $t \in \mathcal{I} \cap \bar{B}(p, 6 f(p) / \varepsilon)$, we have

$$
|p t|-v \leq|p t|-f(p) \leq f(t) \leq V_{(p, w)}(t)=|p t|+w \text {. }
$$

However,

$$
\frac{|p t|+w}{|p t|-w}=1+\frac{2 w}{|p t|-w} \leq 1+\frac{2 w}{3 w / \varepsilon-w} \leq 1+\frac{2 w}{2 w / \varepsilon}=1+\varepsilon,
$$

since $|p t| \geq 6 f(p) / \epsilon \geq 3 w / \varepsilon$. Thus $V_{(p, w)}(t) \leq(1+\varepsilon) f(t)$, for any such $t$.

Lemma 2.6 Let $\mathcal{I}$ be a canvex subset of $\mathbb{R}^{d}, f$ a distance function defined over $I, 0<\varepsilon \leq 1$ a parameter, and $p$ a point in $\mathcal{I}$. Then for any $t \in \overline{\mathcal{I}} \cap B(p, e f(p) / 9)$ and any number $w_{t}$ such that $f(t) \leq w_{t} \leq(1+\varepsilon / 8) f(t)$, we have $f(p) \leq V_{\left(t, w_{t}\right)}(p) \leq(1+\varepsilon) f(p)$.

Proof: Since $|p t| \leq \varepsilon f(p) / 9$, it follows that $f(t) \geq f(p)-$ $|p t| \geq f(p)(1-\varepsilon / 9)$.

Thus,

$$
\frac{\varepsilon f(t)}{8} \geq \frac{\varepsilon}{8} f(p)\left(1-\frac{\varepsilon}{9}\right) \geq \frac{\varepsilon f(p)}{9} \geq|p t|,
$$

implying that $p \in B(t, \varepsilon f(t) / 8)$. By Lemma 2.5, we have $f(p) \leq V_{\left(t, w_{t}\right)}(p) \leq(1+\varepsilon) f(p)$. 
The preceding lemmas suggest the following strategy for constructing an approximation of a distance function $f$ over (a convex portion of) $\mathbb{R}^{d}$ : Pick a point $p$, such that $f(p)$ is close to the global minimum of $f$. The Voronoi diagram $V_{(p, w)}$ approximates $f$ "well" near $p$ and outside a larger ball centered at $p$, where $w$ is an approximation of $f(p)$. We approximate $f$ in the space between those two balls by

partitioning it into concentric spherical shells whose radii form an increasing geometric progression, and by covering each shell by a uniform grid (whose unit length increases with the radius of the shell). In this manner, the number of points needed is only a function of $\varepsilon$ (the approximation factor) and $d$.

When approximating a distance function on a convex subset $\mathcal{I}$ of $\mathbb{R}^{d}$, we have to cope with the possibility that sites might be placed outside $\mathcal{I}$. We overcome this by projecting all such sites onto the boundary of $\mathcal{I}$.

Definition Let $\mathcal{I}$ be a convex subset in $\mathbb{R}^{d}$, and let $x$ be a point in $\mathbb{R}^{d}$. Let $\nu(x, \mathcal{I})$ denote the projection of $x$

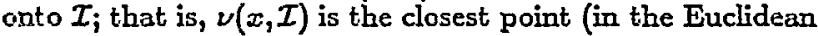
distance) in $\mathcal{I}$ to $x$. Clearly, if $x \in \mathcal{I}$ then $\nu(x, \mathcal{I})=x$.

When $x$ is fixed, we call $\nu(x, \mathcal{I})$ the hook point of $\mathcal{I}$.

Definition Let $r \geq r^{\prime}>0$ be real numbers, let $p$ be a point in $\mathbb{R}^{d}$, and let $\overline{\mathcal{I}}$ be a convex set in $\mathbb{R}^{d}$. We denote by $S\left(p, \mathcal{I}, r, r^{\prime}\right)$ the set $\mathcal{I}_{r^{\prime}} \cap B(p, r) \cap\left(\left(r^{\prime} / \sqrt{d}\right) \mathbb{Z}^{d}\right)$, where $\mathbb{Z}^{d}$ is the integer lattice, and $I_{r}{ }^{\prime}=\cup_{q \in \mathcal{I}} B\left(q, r^{\prime}\right)$ is the set of all point in $\mathbb{R}^{d}$ that are at distance at most $r^{\prime}$ from some point of $\mathcal{I}$. Clearly, $\left|S\left(p, \mathcal{I}, r, r^{\prime}\right)\right|=O\left(\left(r / r^{\prime}\right)^{d}\right)$ (with a constant of proportionality depending on $d)$.

The following technical lemma shows how to pick the sites of the additive weighted Voronoi diagram, so that it $\varepsilon$-approximates a given distance function.

Lemma 2.7 Let $\mathcal{I}$ be a convex subset of $\mathbb{R}^{d}, f: \mathcal{I} \rightarrow \mathbb{R}$ a distance function, $0<\varepsilon \leq 1$ a parameter, $c$ a positive constant, and $p$ a point in $\bar{I}$ such that $f(t) \geq f(p) / c$, for all $t \in \mathcal{I}$. Then one can compute a set $S$ in $\mathcal{I}$ of size $O\left((1 / \varepsilon)^{d} \log (1 / \varepsilon)\right)$ (the constant of proportionality depends on c), such that $p \in S$, and for any weight function $w$ on $S$ satisfying $f(x) \leq w(x) \leq(1+\varepsilon / 8) f(x)$, for all $x \in S$, we have $f(t) \leq V \bar{S}(t) \leq(\overline{1}+\varepsilon) f(t)$, for all $t \in I$, where $\mathcal{S}=(S, w)$.

Proof: Let $w_{p}$ be any number satisfying $f(p) \leq w_{p} \leq$ $(1+\varepsilon / 8) f(p)$.

Let $r_{i}=\left(2^{i}+1\right) w_{p}$, for $i=1, \ldots, m$, where $m=$ $\left\lceil\log _{2}(6 / \varepsilon)\right\rceil$. Let $\mathcal{A}_{1}=B\left(p, r_{1}\right)$, let $\mathcal{A}_{i}=\mathcal{A}\left(p, r_{i-1}, r_{i}\right)$, for $i=2, \ldots, m$, and let $\mathcal{A}_{m+1}=\bar{B}\left(p, r_{m}\right)$. Clearly, $I=$ $U_{i=1}^{m+1}\left(\mathcal{I} \cap \mathcal{A}_{i}\right)$.

Let $r_{1}^{\prime}=\varepsilon w_{p} /(18 c)$, and let $r_{i}^{\prime}=\varepsilon 2^{i-1} w_{p} / 9$, for $i=$ $2, \ldots, m$. Let $S_{i}^{\prime}=\mathcal{A}_{i} \cap S\left(p, \mathcal{I}, r_{i}, r_{i}^{\prime}\right)$, for $i=1, \ldots, m$. Let $S=\{p\} \cup \bigcup_{i=1}^{m} S_{i}$, where $S_{i}=\left\{\nu(x, \mathcal{I}) \mid x \in S_{i}^{\prime}\right\}$, for $i=1, \ldots, m$. See Figure 2 for an illustration of the set $S$.

Let $w$ be any weight function, such that $f(x) \leq w(x) \leq$ $(1+c / 8) f(x)$, for any $x \in S$, and let $\mathcal{S}=(S, w)$.

We claim that $\mathcal{S}$ is the required weighted set. Indeed, let $t \in \mathcal{I}$. If $t \in \mathcal{A}_{m+1}$ then $|p t| \geq\left(2^{m}+1\right) w_{p} \geq 6 f(p) / \varepsilon$. Thus $t \in \bar{B}(p, 6 f(p) / \varepsilon)$ and by Lemmas 2.4 and 2.5 , we heve $f(t) \leq V_{\mathcal{S}}(t) \leq V_{\left(p, w_{p}\right)}(t) \leq(1+\varepsilon) f(t)$.

If $t \in B\left(p, r_{m}\right)$, let $\mathcal{A}_{i}$ be the shell containing $t$. Let $x^{\prime}$ be the closest point to $t$ in $S_{i}^{\prime}$. Let $x=\nu\left(x^{\prime}, \mathcal{I}\right)$. By the definition of $\nu$ and $S_{i}^{\prime}$ and by the convexity of $\mathcal{I}$, we have $|t x| \leq\left|t x^{\prime}\right| \leq r_{i}^{\prime}$ (see Figure 3 ).

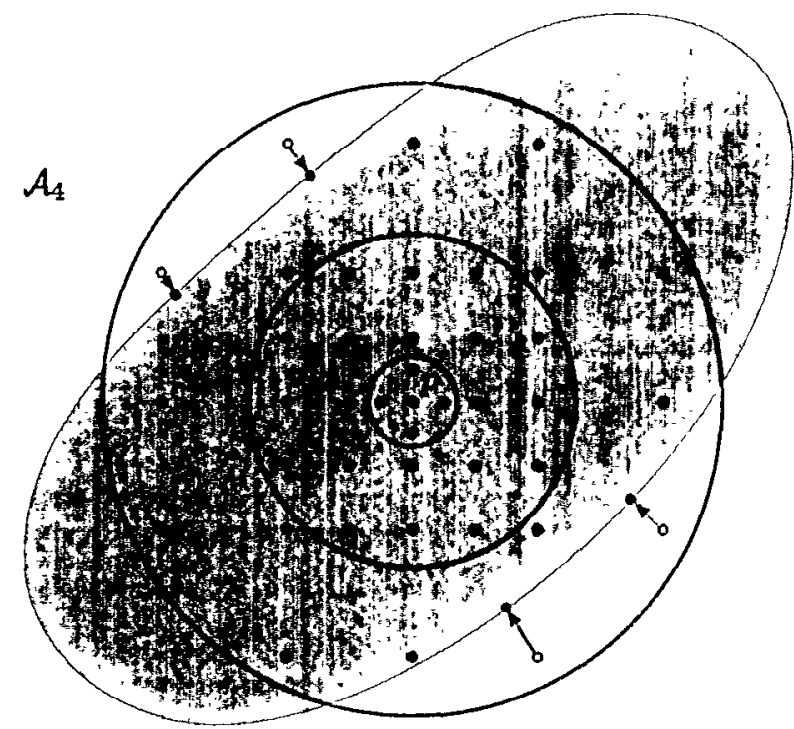

Figure 2: Illustrating the proof of Lemma 2.7. We pick our sites to be on a uniform grid inside each concentric shell around $p$.

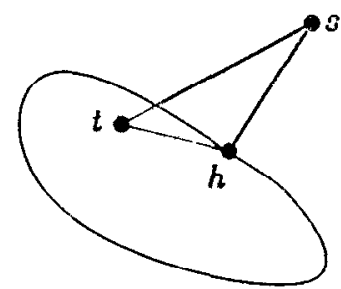

Figure 3: $|s t| \geq|h t|$ for $h=\nu(s, I)$

If $i=1$ then the inequality $f(t) \geq f(p) / c \geq w_{p} / 2 c$ implies that $|t x| \leq r_{1}^{\prime}=\varepsilon w_{p} /(18 c) \leq \varepsilon f(t) / 9$. Thuo $x \in$ $B(t, \varepsilon f(t) / 9)$.

If $i>1$ then we also have $|t x| \leq r_{i}^{\prime}=\varepsilon 2^{i-1} w_{p} / 9 \leq$ $\varepsilon f(t) / 9$, since $f(t) \geq|p t|-f(p) \geq\left(2^{i-1}+1\right) w_{p}-f(p) \geq$ $2^{i-1} w_{p}$. Thus $x \in B(t, \varepsilon f(t) / 9)$.

By Lemma 2.6 and Lemma 2.4, we have $f(t) \leq V_{s}(t) \leq$ $V_{(z, w(z))}(t) \leq(1+\varepsilon) f(t)$.

As for the size of $S$, we have

$$
\begin{aligned}
|S| & =O\left(\sum_{i=1}^{m}\left(\frac{r_{i}}{r_{i}^{l}}\right)^{d}\right) \\
& =O\left(\left(\frac{3 w_{p}}{\varepsilon w_{p} /(18 c)}\right)^{d}+\sum_{i=2}^{m}\left(\frac{\left(2^{i}+1\right) w_{p}}{\varepsilon 2^{i-1} w_{p} / 9}\right)^{d}\right) \\
& =O\left(\frac{1}{\varepsilon^{d}} \log \frac{1}{\varepsilon}\right) .
\end{aligned}
$$

To approximate a distance function $f(\cdot)$ using the constructive proof of Lemma 2.7, we need to find a point which is, within a constant factor, a global minimum of $f$ over the given range. The following lemma shows that this can be easily done if $f$ has a known zero point outside the given range.

Lemma 2.8 Let $\mathcal{I}^{\prime}$ be a subset of $\mathbb{R}^{d}, f: \mathcal{I}^{\prime} \rightarrow \mathbb{R}$ a distanec function, $0<\varepsilon \leq 1$ a parameter, $\mathcal{I}$ a convex subsct of $\mathcal{I}^{\prime}$, 
and a a point in $\mathcal{I}^{\prime} \backslash \mathcal{I}$ such that $f(s)=0$. Then $f(t) \geq$ $f(h) / 2$, for all $t \in I$, where $h=\nu(s, I)$.

Proof: Let $t$ be any point in $\mathcal{I}$. Since $h$ is the closest point in $\mathcal{I}$ to $a$ and $\mathcal{I}$ is convex, it easily follows that $|s t| \geq|h t|$, see Figure 3. Since $f(t)=f(t)+f(s) \geq|s t|$, we have $f(t) \geq|h t|$. Morcover, the segment $h t$ is a contained in $\mathcal{I}$, implying that $f(h) \leq f(t)+|h t| \leq 2 f(t)$.

Remark 2.9 The lemma also holds when $s \in \mathcal{I}$, but then it only yields the trivial bound $f(t) \geq 0$ for all $t \in \mathcal{I}$. Then, of course, $\Delta$ is the required global minimum.

Remark 2.10 Let $\mathcal{I}^{\prime}$ be a set in $\mathbb{R}^{d}$, let $\mathcal{I}$ be a convex subset of $\mathcal{X}^{\prime}, f: \mathcal{I}^{\prime} \rightarrow \mathbb{R}$ a distance function, $0<\varepsilon \leq$ 1 a parameter, and $s$ a point of $\mathcal{I}^{\prime}$ such that $f(s)=0$. Computing a weighted set $\mathcal{S}=(S, w)$ such that the weighted Voronoi diagram induced on $\mathcal{I}$ approximates $f$ up to a factor of $(1+e)$, can be accomplished by following the proof of Lemma 2.7, in four stages:

(i) Compute the point $p=\nu(s, \mathcal{I})$. By Lemma $2.8, p$ is "almost" a minimum of $f$ on $I$.

(ii) Compute an $(\varepsilon / 8)$-approximation $w_{p}$ to $f(p)$ (as prescribed in the lemma).

(iii) Construct the set $S$ of points in $\mathcal{I}$, as prescribed in the proof.

(iv) Approximate the distance function values of all the points of $S$, up to a factor of $1+\varepsilon / 8$, and use these values as the weights for the points of $S$.

Remark 2.11 The set of points $S$ produced in the proof of Lemma 2.7 is made out of $O\left(\log (1 / \varepsilon)\right.$ ) subsets (i.e., $S_{1}, \ldots$, $S_{m}$ ) Buch that $f(x) \leq c f(t)$, for all $x, t \in S_{i}$, for $1 \leq i \leq m$, where $c$ is an appropriate constant. This property enables $\mathrm{WD}$, in the case of ahortest paths on a convex polytope, to approximate the value of the distance function to all the points of $S_{i}$ iimultaneously, yielding a more efficient algorithm. See Remark 3,2 for the details.

Remark 2.12 Let $\mathcal{I}^{\prime}$ be a subset of $\mathbb{R}^{d}$, and let $f, g$ be two diatance functions defined over $\mathcal{I}^{\prime}$. It is easy to verify that $h(x)=\max (f(x), g(x))$ is also a distance function. This implies that the distance function induced by any furthest neighbor Voronoi diagram of a finite set of points in $\mathbb{R}^{d}$, is a distance function in $\mathbb{R}^{d}$. Hence, by Lemma 2.7, we have:

Corollary 2.13 Any furthest neighbor Voronoi diagram of points in $\mathbb{R}^{d}$, can be $\varepsilon$-approximated by a (nearest neighbor) weighted Voronoi diagram, having $O\left(\left(1 / e^{d}\right) \log (1 / \varepsilon)\right)$ sites.

The following is a strengthening of Lemma 2.7, by noticing that in the cases we are going to apply it to, our distance function is the length of a shortest path from a fixed source point to the given point of $\mathcal{I}$. In such cases, if the pource point lies outside $\mathcal{I}$, then a shortest path connecting any point in $\mathcal{I}$ to our source point, must first pass through the boundary $8 I$. We next show that if we are able to $\varepsilon$ approximate the distance on the boundary of $\mathcal{I}$, then we can trivially $\varepsilon$-approximate the distance function to all the pointo of $\mathcal{I}$.

Definition Let $I$ be a convex polytope in $\mathbb{R}^{d}$. We call a function $f: \mathcal{I} \rightarrow \mathbb{R}$ boundary-induced on $\mathcal{I}$, if $f$ is a distance function, and for any $t \in \mathcal{I}$, there exists a point $x \in \partial I$ such that $f(t)=f(x)+|t x|$.

Definition Given a convex polytope $I$ in $\mathbb{R}^{d}$, we denote by $\phi(\mathcal{I})$ the set of all the facets $((d-1)$-faces $)$ of $\mathcal{I}$.

Lemma 2.14 Let $\mathcal{I}$ be a convex polytope in $\mathbb{R}^{d}, f: \mathcal{I} \rightarrow$ $\mathbb{R}$ a boundary-induced distance function, and $0<\varepsilon \leq 1$ a parameter. For any facet $F$ of $\mathcal{I}$, let $\mathcal{S}(F)=\left(S_{F}, w_{F}\right)$ be a weighted set of points in $F$, such that $f(t) \leq V_{\mathcal{S}(F)}(t) \leq$ $(1+\varepsilon) f(t)$, for all $t \in F$. Then $f(t) \leq V_{s}(t) \leq(1+\varepsilon) f(t)$, for all $t \in \mathcal{I}$, where $\mathcal{S}=(S, w)=\cup_{F \in \phi(\mathcal{I})} \mathcal{S}(F)$.

Proof: For any $t \in I$, let $x$ be the point in $\partial \mathcal{I}$ satisfying $f(t)=f(x)+|t x|$, and let $F$ be a facet of $\mathcal{I}$ that contains x. By Lemma 2.4, we have

$$
\begin{aligned}
f(x)+|t x| & =f(t) \leq V_{s}(t) \leq V_{s}(x)+|t x| \\
& \leq V_{s(F)}(x)+|t x| \leq(1+\varepsilon) f(x)+|t x| \\
& \leq(1+\varepsilon)(f(x)+|t x|)=(1+\varepsilon) f(t) .
\end{aligned}
$$

Remark 2.15 Let $\mathcal{I}^{\prime}$ be a set in $\mathbb{R}^{d}$, let $\mathcal{I}$ be a convex subset of $\mathcal{I}^{\prime}, f: \mathcal{I}^{\prime} \rightarrow \mathbb{R}$ a boundary-induced distance function, $0<\varepsilon \leq 1$ a parameter, and $s$ a point of $\mathcal{I}^{\prime}$ such that $f(s)=0$. Computing a weighted set $\mathcal{S}=(S, w)$ such that the veighted Voronoi diagram induced on $I$ approximates $f$ up to a factor of $(1+\varepsilon)$, can be done by applying the algorithm described in Remark 2.10 for each facet of $\mathcal{I}$. By Lemma 2.14, the union of all these veighted sets has the required properties.

\section{Approximate Shortest-Path Map on a Polyhedral Surface in $\mathbb{R}^{3}$}

Let $\mathcal{P}$ be a given polyhedral surface in $\mathbb{R}^{3}$ with $n$ edges, let $s$ be a source point on $\mathcal{P}$, and let $0<\varepsilon \leq 1$ be a given parameter. In this section, we give an algorithm for constructing an approximation map on $\mathcal{P}$ of complexity $O((n / e) \log (1 / e))$, such that given any $t \in \mathcal{P}$, one can compute in $O(\log (n / \varepsilon))$ time a distance $\Delta_{\mathcal{P}}(s, t)$ satisfying $d_{\mathcal{P}}(s, t) \leq \Delta_{\mathcal{P}}(s, t) \leq$ $(1+\varepsilon) d_{\mathcal{P}}(s, t)$.

Although the following description is rather technical, one has to bear in mind that it is a straightforward implementation of the technique of Section 2. Namely, for each edge of our domain (polyhedral terrain, or a convex polytope) ve compute a "small" set of points, we approximate the (geodesic) distance from the source point to all those points, and we construct the veighted additive Voronoi diagram that those points induce on each face of the domain.

Definition A polyhedral surface $\mathcal{P}$ in $\mathbb{R}^{3}$ is the union of a collection of planar polygonal faces, with their edges and vertices, such that each edge is incident to at most two faces and any pair of faces intersect either at a common edge, a common vertex, or not at all. A face is a simple closed polygon (i.e., it contains its boundary), and an edge is a closed segment (i.e., it contains its endpoints). Without loss of generality we assume that all the faces are triangular (since simple polygons may be triangulated in linear time [4] and the number of new edges introduced by the triangulation is linear in the number of vertices). We also assume that $\mathcal{P}$ is connected. 
A polyhedral terrain is a polyhedral surface that intersects every vertical line in at most a single point.

Definition Given a polyhedral surface $\mathcal{P}$ in $\mathbb{R}^{3}$, and any two points $s, t$ on $\mathcal{P}$, we denote by $d_{\mathcal{P}, s}(t)$ the length of a shortest path between $s$ and $t$ on $\mathcal{P}$.

As noted in Example $2.2(\mathrm{i}), d_{P_{1},}(\cdot)$ is a distance function on $\mathcal{P}$. Moreover, if $F$ is a face of $\mathcal{P}$ and $s \notin F$ then $d_{\mathcal{P}, s}$ is boundary induced on $F$. (If $s \in F$ then $d_{\mathcal{P}_{8},}(t)$ is the Euclidean distance $|s t|)$

The following theorem is the main result of this section.

Theorem 3.1 Let $\mathcal{P}$ be a polyhedral surface in $\mathbb{R}^{3}$ with $n$ cdges, $s$ a source point on $\mathcal{P}$, and $0<\varepsilon \leq 1$ a real parameter. Then there exists a subdivision II of $\mathcal{P}$ of compleaity $\mathcal{O}((n / c) \log (1 / \varepsilon))$, which facilitates $\varepsilon$-approximate shortest path queries from $s$ on $\mathcal{P}$. That is, for any query point $t$ on $\mathcal{P}$, one can compute, in $O(\log (n / \varepsilon))$ time, a distance $\Delta_{\mathcal{P}}(s, t)$, such that $d_{\mathcal{P}_{, s}}(t) \leq \Delta_{\mathcal{P}}(s, t) \leq(1+\varepsilon) d_{\mathcal{P}, s}(t)$.

The map can be computed in $O\left(n^{2} \log n+(n / \varepsilon) \log (1 / \varepsilon)\right.$ $\log (n / \varepsilon))$ time, if $\mathcal{P}$ is an arbitrary polyhedral surface, ond in $O\left(\left(n / \varepsilon^{3}\right) \log (1 / \varepsilon)+\left(n / \varepsilon^{1.5}\right) \log (1 / \varepsilon) \log n\right)$ time, if $\mathcal{P}$ is a convex polytope.

The space used by the algorithm is $O((n / \varepsilon) \log (1 / \varepsilon))$, if $\mathcal{P}$ is cither a convex polytope or a polyhedral terrain, and $O\left(n^{2}+(n / \varepsilon) \log (1 / \varepsilon)\right)$ otherewise.

Proof: For each face $F$ of $\mathcal{P}$ that does not contain $s$, we construct a weighted Voronoi diagram that approximates $d_{P, \text { on }} F$. By Remark 2.15, this can be done by constructing a weighted Voronoi diagram on each edge of $\mathcal{P}$, as outlined in Rernark 2.10 .

For the general case, we compute the exact shortest-path map of $s$ on $\mathcal{P}$, using the algorithm of [16], in $O\left(n^{2} \log n\right)$ time. The exact map enables us to compute the shortest distance from $s$ to any point of $\mathcal{P}$ in $O(\log n)$ time. Thus, computing the distances from $s$ to the $n$ hooks of the edges of $\mathcal{P}$ takes additional $O(n \log n)$ time. The hook point of an edge is the closest point of the edge to $s$, and it can be computed in $O(1)$ time.

For the convex case, we approximate the distances on $\mathcal{P}$ to all the hooks on the edges of $\mathcal{P}$, up to a factor of $(1+\varepsilon / 8)$. This takes $O\left(n / \varepsilon^{3}+\left(n / \varepsilon^{1.5}\right) \log n\right)$ time, using the algorithm of $[1$, Sec. 6].

For each edge $\varepsilon$ of $\mathcal{P}$, we compute a set $S_{e}$ of $O((1 / \varepsilon)$ $\log (1 / \varepsilon))$ points on $e$, as specified in the proof of Lemma 2.7, taking the corresponding $p$ to be the hook of $e$ and $w_{p}$ to be the approximated (exact in the non-convex case) distance along $\mathcal{P}$ from $s$ to $p$. Let $S=\cup_{e} S_{e}$, taken over all edges $e$ of $\mathcal{P}$.

We now compute (or approximate) the distances from $s$ to all the points in $S$. For the non-convex case, this can be done in $O((n / \varepsilon) \log (1 / \varepsilon) \log n)$ time, using the exact shortest path map.

For the convex case, we compute approximate distances from $s$ to all points of $S$, up to a factor of $(1+\varepsilon / 8)$. Using the observation of Remark 2.11, we partition $S$ into $O(n \log (1 / \varepsilon))$ sets, each of size $O(1 / \varepsilon)$, such that the required distances to the points in each such set are within a fixed constant factor of each other (namely, for each edge $\epsilon$ of $\mathcal{P}$, the set $S_{e}$ is decomposed into $O(\log (1 / \varepsilon))$ sets, as in the proof of Lemma 2.7). Using the algorithm described in Remark 3.2 below, we can compute the distances from $s$ to all the points of $S$ in $O\left(\left(n / \varepsilon^{3}\right) \log (1 / \varepsilon)+\left(n / \varepsilon^{1.5}\right) \log (1 / \varepsilon) \log n\right)$ time.
Next, we compute, for each face $F$ of $\mathcal{P}$, the weighted Voronoi diagram induced by the weighted points of $\mathcal{S}$ that lie on $\partial F$. This takes $O\left((n / \varepsilon) \log ^{2}(1 / \varepsilon)\right.$ ) overall time (see $[10])$, since each face contains $O((1 / \varepsilon) \log (1 / \varepsilon))$ points of $\mathcal{S}$. Let $I$ be the resulting map, consisting of the union of all those facial Voronoi diagrams.

By Lemma 2.7 and Lemma 2.14, the map II on $\mathcal{P}$ has the required properties. Moreover, we can preprocess each face $F$ of $\mathcal{P}$ in $O\left((1 / \varepsilon) \log ^{2}(1 / \varepsilon)\right)$ time, such that point location queries on $F$ can be answered in $O(\log (1 / \varepsilon))$ time (sce [18]). Overall, this preprocessing takes $O\left((n / \varepsilon) \log ^{2}(1 / \varepsilon)\right)$ time.

To answer a approximate shortest path query for a query point $q$, the algorithm must locate the face of $\mathcal{P}$ containing $q$. If $\mathcal{P}$ is a polyhedral terrain, we project the terrain into the $x y$-plane and preprocess it, in $O(n \log n)$ time, for planar point location. If $\mathcal{P}$ is a convex polytope, it can be preprocessed in linear time to answer point location queries in $O(\log n)$ time (see [9]). Otherwise, we preprocess $\mathcal{P}$ for spatial point-location in $O\left(n^{2} \log n\right)$ time, and $O\left(n^{2}\right)$ opnce, with $O(\log n)$ query time, using the algorithm of [23].

Given any query point $q$ on $\mathcal{P}$, the algorithm computes the face $F$ of $\mathcal{P}$ that contains $q$ in $O(\log n)$ time. Locating the face of the subdivision II that contains $q$ takes an additional $O(\log (1 / \varepsilon))$ time. Thus, $\varepsilon$-approximate shortest path queries for $\mathcal{P}$ can be answered in $O(\log (n / \varepsilon))$ time. (If the face containing $q$ is already known, the query time reduced to $O(\log (1 / \varepsilon))$.)

Definition Let $P$ be a convex body in $\mathbb{I R}^{3}$. An outer path of $P$ is a curve $\gamma$ connecting two points on $\partial P$ and digjoint from the interior of $P$.

Remark 3.2 Let $P$ be a convex polytope in $\mathbb{R}^{3}$, a a nource point on $P, T$ a set of points on $P$, and $0<\varepsilon S 1$ a prescribed parameter. One can e-approximate the length of the shortest path from $s$ to all the points of $T$ on $P$, in $O\left((n+|T|) / \varepsilon^{3}+\left((n+|T|) / \varepsilon^{1.5}\right) \log (n+|T|)\right)$ time, by adding the points of $T$ as vertices to $P$ and by using the algorithm of $[1$, Sec. 6$]$.

The algorithm of [1] works by computing an approximntion polytope for each point of $T$, and by computine the exact distance from $s$ to the point on this polytope.

Moreover, suppose that $T$ can be partitioned into $m$ sets $T_{1}, \ldots, T_{m}$, such that $d_{P_{1},}(t) \leq c \cdot d_{P_{1} s}\left(t^{\prime}\right)$, for all $t, t^{\prime} \in T_{\mathbf{i}}$ and for each $i=1, \ldots, m$, where $c$ is a prescribed constant, and all the points of $T_{i}$ belong to the same edge of $P$, for any fixed $i=1, \ldots, m$. Then it is possible to speed up the above algorithm, as follows. Instead of constructing an approximation polytope for each destination point separately, we construct an approximation polytope that can be used to approximate the distances from $s$ to all the points of $T_{i}$, for $i=1, \ldots, m$. This is done by ensuring that all the pointo of $T_{i}$ lie on the boundary of the approximation polytope calculated by the algorithm, which can be enforced by intersecting it with a supporting plane of $P$ pnosing through the edge containing the points of $T_{i}$ (adding at most one new face to the approximation polytope). We also necd to use a more refined approximation polytope, so as to achieve the claimed error bound, but since $c$ is a constant this does not change the asymptotic cornplexity of the algorithm. See [1] for the technical details.

This improves the running time to

$$
O\left(n+\frac{m}{\varepsilon^{3}}+\frac{m}{\varepsilon^{1.5}} \log n+\frac{|T|}{\varepsilon^{1.5}}\right)
$$


by constructing an approximation polytope for the points of $T_{i}$ (in $O\left(\frac{m}{6} \log n\right)$ time), computing the exact distance map from the source point on the approximation polytope (in $O\left(1 / e^{3}\right)$ time), and extracting the shortest path to each point of $T_{i}$, repeating all this, for $T_{1}, \ldots, T_{m}$. Moreover, for each point $t \in T_{i}$, the algorithm computes a polygonal outer path of $P$, made out of $O\left(1 / e^{1.5}\right)$ segments, that realizes the approximated distance.

Remark 3.3 The algorithm of [16] works for arbitrary polyhedral surfaces; in particular, it is not restricted to polyhedral terrains. Thus, the algorithm of Theorem 3.1 also works for general polyhedral surfaces.

Remark 3.4 For a convex polytope $P$ with $n$ edges in $\mathbb{R}^{3}$, one can compute an approximation map that can be used to compute an outer path that realizes the approximate distance. This is done by modifying the algorithm of Theorem 3.1, such that it atores an outer path from the source point to each of the constructed sites, where the outer path realizen ita $e$-approximate distance. Such a path is readily available from the procedure used to compute the approximate dintance to the site, and the complexity of such a path is $O\left(1 / e^{1,5}\right)$ (See [1]). The space needed to store the extended approximation map is $O\left(n / e^{2,5} \log (1 / e)\right)$, and the computation time remains $O\left(\left(n / e^{3}\right) \log (1 / \varepsilon)+\left(n / e^{1.5}\right) \log (1 / \varepsilon)\right.$ $\log n$ ).

The new map can be used to answer approximate shortent path queries, in $O(\log (n / \varepsilon))$ time, and also compute, in additional $O\left(1 / e^{1,5}\right)$ time, an outer path of the convex polytope realizing this distance. Such an outer path can be projected onto the boundary of the convex polytope, in additional $O\left(n \log (1 / \varepsilon)+1 / e^{3}\right)$ time, resulting in a path on $\theta P$ which in an $e$-approximation to the shortest path, sec [1]. Note however that the performance of the enhanced deta structure is poorer both in terms of storage and query time.

\section{Constructing Spatial Approximate Shortest-Path Maps in $\mathbb{R}^{3}$}

Let $\mathcal{O}$ be a collection of pairwise-digjoint polyhedral obstacles in $\mathbb{R}^{3}, 8$ a source point in $\mathbb{R}^{3} \backslash$ int $\bigcup \mathcal{O}$, and $0<\varepsilon \leq 1$ a parameter. In this section, we present an algorithm for preprocesaing $\mathcal{O}$ such that for any point in $\mathbb{R}^{3}$ (or, more preciuely, for any 'free' point that can be reached from $s$ without penetrating into an obstacle) one can compute, in $O(\log (n / \varepsilon))$ time, a distance $\Delta_{O, a}(t)$ satisfying $d_{0, s}(t) \leq$ $\Delta_{0,0}(t) \leq(1+e) d_{0, \alpha}(t)$, where $d_{0,0}(t)$ is the length of a ohortest path between $s$ and $t$ that avoids the interiors of the obntacles.

The preprocessing time of the algorithm is roughly $O\left(n^{4} / e^{6}\right)$, which show that the problem of approximating the distance from a single source to all the 'free' points in $\mathbb{R}^{3}$, in not much harder (computationally) than approximating the distance between any specific pair of points (which can be done in roughly $O\left(n^{2} / e^{1}\right)$ time, see [7]). In fact, for a flxed nource point and many destination points, our algorithm will actually be faster. The problem of computing the exact distance between two points in $\mathbb{R}^{3}$ among polyhedral obatacles is NP-hard, as shown by Canny and Reif [3], and the fastest available algorithms for this problem run in time that is exponential in the total number of obstacle vertices $[20,21,22]$.

Deflnition Let $\mathcal{O}$ be a collection of pairwise-disjoint polyhedral obstacles with a total of $n$ edges in $\mathbb{R}^{3}$, and $s$ a source point in $F P(\mathcal{O})=\mathbb{R}^{3} \backslash$ int $\bigcup_{O \in \mathcal{O}} O$. Let $F P(\mathcal{O}, s)$ denote the set of all points in $F P(\mathcal{O})$ that can be connected to $s$ by a path that avoids the interiors of the obstacles of $\mathcal{O}$.

For any $t \in F P(\mathcal{O}, s)$, we denote, as above, by $d_{0, s}(t)$ the length of a shortest path between $s$ and $t$, that avoids the interiors of the obstacles of $\mathcal{O}$.

As noted in Example 2.2 (ii), $d_{O, s}(\cdot)$ is a distance function over $F P(\mathcal{O}, s)$, and for any convex set $\mathcal{I} \subseteq F P(\mathcal{O}, s)$ such that $s \notin \mathcal{I}$, the function $d_{O,},(\cdot)$ is boundary induced over $\mathcal{I}$.

Theorem 4.1 (Clarkson [7]) Given a set $\mathcal{O}$ of polyhedral obstacles in $\mathbb{R}^{3}$, and points $s$ and $t$, an e-approximate path between $s$ and $t$ that does not penetrate into any obstacle in $\mathcal{O}$ can be computed in

$$
O\left(\frac{n^{2}}{\varepsilon^{4}} \beta(n) \log \frac{n}{\varepsilon}+n^{2} \log (n \rho) \log (n \log \rho)\right)
$$

time, where $n$ is the number of obstacle edges, and $\rho$ is the ratio of the length of the longest edge in $\mathcal{O}$ to the Euclidean distance between $s$ and $t, \beta(n)=\alpha(n)^{O(\alpha(n))^{\circ(1)}}$, and $\alpha(n)$ is the inverse of the Ackermann function.

The following theorem is the main result of this section.

Theorem 4.2 Let $\mathcal{O}$ be a collection of pairwise-disjoint polyhedral obstacles with $n$ edges in $\mathbb{R}^{3}, s$ as source point in $F P(\mathcal{O})$, and $0<\varepsilon<1$ a parameter. Then a subdivision $\mathcal{M}$ of $F P(\mathcal{O}, s)$ of complexity $O\left(n^{2} / e^{4+\delta}\right)$, for any $1>\delta>0$, can be computed in

$$
O\left(\frac{n^{4}}{\varepsilon^{2}}\left(\frac{\beta(n)}{\varepsilon^{4}} \log \frac{n}{\varepsilon}+\log (n \rho) \log (n \log \rho)\right) \log \frac{1}{\varepsilon}\right)
$$

time, where $\rho$, and $\beta(n)$ are as above.

For any query point $t \in F P(\mathcal{O}, s)$, one can compute in $O(\log (n / \varepsilon))$ time a distance $\Delta_{0,8}(t)$, such that $d_{0,0}(t) \leq$ $\Delta \Delta_{0, s}(t) \leq(1+\varepsilon) d_{0, s}(t)$.

Proof: First, we partition $F P(\mathcal{O})$ into $O\left(n^{2}\right)$ vertical prisms. This can be easily done by erecting a vertical wall from each edge of the obstacles. For an edge $e$ of the obstacles, such a wall is the set of all points in $F P(\mathcal{O})$ that lie on vertical rays emanating from the edge, and not intersecting the obstacles. Let $\mathcal{M}^{\prime \prime \prime}$ denote the resulting partition of $F P(\mathcal{O})$. It is easy to verify that the complexity of $\mathcal{M}^{\prime \prime \prime}$ is $O\left(n^{2}\right)$, and that it can be computed in $O\left(n^{2} \log n\right)$ time.

We refine $\mathcal{M}^{\prime \prime \prime}$, by further partitioning each cell of $\mathcal{M}^{\prime \prime \prime}$ into vertical triangular prisms. This is done by projecting each cell of $\mathcal{M}^{\prime \prime \prime}$ into the $x y$-plane, and by triangulating the resulting polygon, in $O(m \log m)$ time (sce [18]), where $m$ is the number of vertices of the polygon. For each new edge created, we erect a corresponding vertical wall inside the cell. Let $\mathcal{M}^{\prime \prime}$ be the resulting subdivision of $F P(\mathcal{O})$. Clearly, the complexity of $\mathcal{M}^{\prime \prime}$ remains $O\left(n^{2}\right)$, and it can be computed in additional $O\left(n^{2} \log n\right)$ time.

Let $T_{v}$ be the vertical prism in $\mathcal{M}^{\prime \prime}$ that contains $s$. We construct an adjacency graph $G$ on the vertical prisms of $\mathcal{M}^{\prime \prime}$. By computing the connected component of $G$ that contains $T_{v}$, one obtains the subdivision $\mathcal{M}^{\prime}=\mathcal{M}^{\prime \prime} \cap F P(\mathcal{O}, s)$,

Each cell $\mathcal{I}$ of $\mathcal{M}^{\prime}$ is a vertical prism, having at most 5 faces. We can approximate the distance function $d_{0,0}(t)$ inside $\mathcal{I}$ by computing a weighted set $\mathcal{S}_{\mathcal{I}}=\left(S_{I}, w_{I}\right)$, as specified in the proofs of Lemma 2.7 and Lemma 2.14. To do so, it is necessary to $(\varepsilon / 8)$-approximate the value of $d_{0,0}(\cdot)$ 
for $O\left(\left(1 / \varepsilon^{2}\right) \log (1 / \varepsilon)\right)$ points (i.e., the points of $\left.\mathcal{S}_{\mathcal{I}}\right)$. By Theorem 4.1, this takes

$$
O\left(\left(\frac{\beta(n)}{\varepsilon^{4}} \log \frac{n}{\varepsilon}+\log (n \rho) \log (n \log \rho)\right) \frac{n^{2}}{\varepsilon^{2}} \log \frac{1}{\varepsilon}\right)
$$

time. The weighted Voronoi diagram $\mathcal{V}_{S_{\mathcal{I}}}$ induced by $S_{\mathcal{I}}$ inside $\mathcal{I}$ approximates $d_{\mathcal{O}}$, inside $I$ up to a factor of $1+\varepsilon$.

Let $\mathcal{S}=\cup_{\mathcal{I} \in \mathcal{M}^{\prime}} \mathcal{S}_{\mathcal{I}}$. Clearly, one can $(\varepsilon / 8)$-approximate the distance between $s$ and all the sites of $\mathcal{S}$ in

$$
O\left(\frac{n^{4}}{\varepsilon^{2}}\left(\frac{\beta(n)}{\varepsilon^{4}} \log \frac{n}{\varepsilon}+\log (n \rho) \log (n \log \rho)\right) \log \frac{1}{\varepsilon}\right)
$$

time.

Let $\mathcal{M}$ be the subdivision $U_{I \in \mathcal{M}^{\prime}}\left(\mathcal{V}_{s_{\mathcal{I}}} \cap \mathcal{I}\right)$. We preprocess $\mathcal{M}$ for spatial point location, by constructing a two-level spatial point location data structure. First, we preprocess $\mathcal{M}^{\prime}$ for point location in $O\left(n^{2} \log n\right)$ time, using the algorithm of [23]. Next, we preprocess each cell $\mathcal{I}$ of $\mathcal{M}^{\prime}$ for nearest neighbor queries for the weighted set $\mathcal{S}_{\mathcal{I}}$. By Lemma 4.3 below, performing this preprocessing for all the cells of $\mathcal{M}^{\prime}$ takes a total of $O\left(n^{2} / \varepsilon^{4+\delta}\right)$ randomized expected time and space, for any $\delta>0$.

For any query point $t \in F P(\mathcal{O}, s)$, we can compute in $O(\log n+\log (1 / \varepsilon))=O(\log (n / \varepsilon))$ time, the cell of $\mathcal{M}$ that contains $t$; that is, in $O(\log (n / \varepsilon))$ time, one can compute a distance $\Delta_{O_{, \delta}}(t)$, such that $d_{0,8}(t) \leq \Delta_{\mathcal{O}_{8},}(t) \leq(1+$ c) do,a $(t)$.

To complete the description and analysis of the algorithm, we next show how to preprocess a weighted set in $\mathbb{R}^{3}$ so that one can perform efficient nearest neighbor queries in the additive weighted Voronoi diagram that it induces.

Definition Let $\mathcal{S}=(S, w)$ be a weighted set in $\mathbb{R}^{3}$. We decompose the weighted Voronoi diagram $\mathcal{V}_{s}$ into "simpler" cells in the following way: For each cell $C$ in $\mathcal{V}_{s}$, we compute the spherical map $S_{C}$ of the cell, by projecting the boundary of the cell onto the sphere of directions centered at $p_{C}$, where $p_{C}$ is the site of $C$ in $S$. (We use here the well-known property that $C$ is star-shaped with respect to $p_{C}$.) We decompose $S_{c}$ into pseudo-vertical subcells on the sphere of directions, by drawing a meridian arc upwards and downwards from each vertex $S_{c}$, and from each locally longitudeextremal point on any arc of $S_{c}$, and by extending each of these meridian ares until it hits another arc of $S_{c}$ or, failing this, all the way to the poles of the sphere of directions. Clearly, the complexity of $\mathrm{S}_{\mathrm{c}}$ is linear in the complexity of the cell $C$.

We project each "vertical" trapezoid in $\mathrm{S}_{\mathrm{c}}$ back into $C$, to obtain the portion within $C$ of the cone with apes: $p C$ spanned by the trapezoid. This defines a decomposition of $C$ into simple subcells, such that each subcell is uniquely defined by at most 6 points of $S$. We decompose all the cells of $\mathcal{V}_{S}$ in a similar manner, and let $\mathcal{C}(\mathcal{S})$ denote the resulting subdivision. We call $\mathcal{C}(\mathcal{S})$ the spherical decomposition of $\mathcal{V}_{s}$.

For a weighted set $\mathcal{R} \subseteq \mathcal{S}$ and a subcell $\mathcal{T} \in \mathcal{C}(\mathcal{R})$, a weighted point $\left(p, w_{p}\right) \in \mathcal{S}$ conflicts with $\mathcal{T}$ if there esists a point $t \in \mathcal{T}$, such that $V_{\left(p, w_{p}\right)}(t)<V_{\mathcal{R}}(t)$. Let $K(\mathcal{S}, \mathcal{T})$ denote the set of all the points of $\mathcal{S}$ that conflict with $\mathcal{T}$. The confict size of $\mathcal{T}$ is $w(\mathcal{S}, \mathcal{T})=|K(\mathcal{S}, \mathcal{T})|$.

Lemma 4.3 Let $\mathcal{S}=(S, w)$ be a weighted set of $m$ points in $\mathbb{R}^{3}$, and $\delta>0$ be a parameter. Onc can compute, in $O\left(m^{2}+\delta\right)$ randomized expected time, a data structure for ncarest-neighbor queries, of size $O\left(m^{2+\delta}\right)$, such that for any point $p \in \mathbb{R}^{3}$, one can compute, in $O(\log m)$ time, the cell of $\mathcal{V}_{s}$ that contains $p_{\text {; that }}$ is, the point in $\mathcal{S}$ realizing the distance $V_{s}(p)$.

Proof: We construct the data-structure using a randomized divide and conquer algorithm. We randomly pick a subset $R$ of $S$ of size $r$, where $r$ is a parameter to be specified latcr. One can compute the weighted Voronoi diagram of $\mathcal{R}=(R, w)$, in $O\left(r^{2}\right)$ time, by Remark 2.3, and construct the spherical decomposition $\mathcal{C}(\mathcal{R})$ in $O\left(r^{2} \log r\right)$ additional time, using plane sweeping techniques on the sphere of directions (see [18]).

For each subcell $\mathcal{T}$ in $\mathcal{C}(\mathcal{R})$, we compute its conflict size $w(\mathcal{S}, \mathcal{T})$. Each subcell in $\mathcal{C}(\mathcal{R})$ is uniquely defined by at most 6 sites in $\mathcal{R}$, and if $K(\mathcal{S}, \mathcal{T}) \cap \mathcal{R} \neq \emptyset$ then $\mathcal{T} \notin C(\mathcal{R})$. We can thus apply the analysis of Clarkson and Shor. By $[8$, Corollary 3.8], $w(\mathcal{S}, \mathcal{T}) \leq c \cdot(n \log r) / r$, for all $\mathcal{T} \in \mathcal{C}(\mathcal{R})$, with probability at least $1 / 2$, where $c>0$ is an eppropriate constant. We sample $\mathcal{R}$ from $\mathcal{S}$ repeatedly until we get a sample that fulfills this condition. Overall, this stage takes $O\left(m r^{2}+r^{2} \log r\right)$ expected running time. For each cell $T \in$ $\mathcal{C}(\mathcal{R})$, we construct recursively a data-structure for pointlocation in the Voronoi diagram $\mathcal{V}_{k(S, \pi)}$.

For any query point $p$, locating the subcell $\mathcal{T}$ in $\mathcal{C}(\mathcal{R})$ that contains $p$ is done by a brute force search inside $\mathcal{C}(\mathcal{R})$, in $O\left(r^{2}\right)$ time. Then, we compute the point realizing $V_{\mathcal{S}}(p)$ by recursively performing a nearest neighbor query in the data-structure computed for $\mathcal{V}_{K(}(s, \pi)$. Thus, a query takes $Q(m)=Q(c(m \log r) / r)+O\left(r^{2}\right)$ time, and the datastructure can be computed, in randomized expected tirne

$$
T(m)=T(r)+O\left(r^{2}\right) T\left(\frac{c m \log r}{r}\right)+O\left(m r^{2}+r^{2} \log r\right) .
$$

Choosing $r$ to be a sufficiently large constant, we have $Q(m)$ $=O(\log m)$, and $T(m)=O\left(m^{2+\delta}\right)$ (where the constants of proportionality depend on $\delta$ ). A similar bound bolds for the space required by the algorithm.

Remark 4.4 The only stage in the algorithm of Theorem 4.2 that uses randomization is the construction of the spatiol point-location data described in Lemma 4.3. This can be replaced by a deterministic data-structure as follows.

We observe that each spherical cell, in the decomposition described above, can be parameterized by 24 parameters ( 6 sites and their respective weights). Thus, we define a range space $(\mathcal{S}, \mathfrak{R})$, where $\mathfrak{R}$ is the set of all possible subsets of $\mathcal{S}$ that are contained inside such a spherical cell. It is easy to verify that this is a range space having finite VC-dimension. By a result of Matoušek [15], we can compute, in $O\left(m r^{O(1)}\right)$ time, a subset $\mathcal{R}$ of $\mathcal{S}$ having $O(r \log r)$ points, which is $(1 / r)$-net of $(\mathcal{S}, \mathfrak{R})$. In particular, the set $\mathcal{K}$ can replace the random sample in the proof of Lemma 4.3, see [15]. This yields a deterministic algorithm with the same time/opace complexity as in Lemma 4.3.

Alternatively, one can naively preprocess the Voronoi diagram $\mathcal{V}_{S}$ for spatial point-location directly, see [23]. However, this approach is considerably less efficient than the approach proposed above.

\section{Conclusions}

In this paper we have presented two results for computing approximate maps that facilitate shortest paths queries on the surface of a convex polytope or on a polyhedral surface in 3-space, or among polyhedral obstacles in 3-space. We conclude by mentioning the following open problems. 
- Can an e-approximate shortest path between two points on a polyhedral terrain, or on the surface of a nonconvex polyhedron, be computed in time that is near-linear in the number of edges? A recent subquadratic solution has been obtained by Varadarajan and Agarwal [24], but it only computes a constantfactor approximation to the shortest path.

- Can the exact shortest path between two points on a convex polyhedron be computed in near-linear time? in aubquadratic time?

- Can the methods and techniques used in this paper be extended to handle shortest path queries for weighted ourfaces (as in $[13,14])$ ?

\section{Acknowledgments}

The author wisheв to thank Pankaj Agarwal and Micha Sharir for helpful discussions concerning the problems studied in this paper and related problems. Micha Sharir has also ouggested Lemma 4.3. The author also wishes to thank the referees for their comments and auggestions.

\section{References}

[1] Agarwal, P., Har-Peled, S., Sharm, M., aNd Varadarajan, K. R. Approximate shortest paths on a convex polytope in three dimensions. J. Assoc. Comput. Mach. \&4 (1997), 567-584.

[2] Aurenhammen, F. Voronoi diagrams: A survey of a fundamental geometric data structure. ACM Comput. Surv. 29 (1991), 345-405.

[3] Canny, J., and ReIF, J. H. New lower bound techniques for robot motion planning problems. In Proc. 88th Annu. IEEE Sympos. Found. Comput. Sci. (1987), pp, 49-60.

[1] Chazelle, B. Triangulating a simple polygon in linear time. Discrete Comput. Geom. 6 (1991), 485-524.

[6] Cinen, J., AND HaN, Y. Shortest paths on a polyhedron; Part I: computing shortest paths. Int. J. Comput. Geom, \& Appl, 6, 2 (1996), 127-144.

[0] Chor, J., Sellen, J., AND YAP, C. K. Approximate Buclidean shortest path in 3-space. In Proc. 10th Annu. ACM Sympos. Comput. Geom. (1994), pp. 41-48.

[7] Clarkson, K. L. Approximation algorithms for shortest path motion planning. In Proc. 19th Annu. ACM Sympos, Theory Comput. (1987), pp. 56-65.

[8] Clankson, K. L., and SHOR, P.W. Applications of random sampling in computational geometry, II. $D_{\text {is- }}$ crete Comput. Geom. \& (1989), 387-421.

[9] DobKIn, D. P., AND KmKPATRICK, D. G. A linear algorithm for determining the separation of convex polyhedra. J. Algorithms 6 (1985), 381-392.

[10] Fonturse, S. J. A sweepline algorithm for Voronoi diagrams. Algorithmica 2 (1987), 153-174.

[11] HAR-PELED, S. Approximate shortest paths and Geodesic diameters on convex polytopes in three dimensions. In Proc. 19th Annu. ACM Sympos. Comput. Geom. (1997), pp. 359-365.

[12] Hershberger, J., ANd SuRI, S. Practical methods for approximating shortest paths on a convex polytope in $\mathbb{I}^{\mathrm{s}}$. In Proc, 6th ACM-SIAM Sympos. Discrete Algorithms (1995), pp. 447-456.
[13] LanthiER, M., Mameshwari, A., AND SaCK, J.-R. Approximating weighted shortest paths on polyhedral surfaces. In Proc. 13th Annu. ACM Sympos. Comput. Geom. (1997), pp. 274-283.

[14] MATA, C., AND MrTChelL, J. A new algorithm for computing the shortest paths in planar subdivisions. In Proc. 13th Annu. ACM Sympos. Comput. Geom. (1997), pp. 264-273.

[15] MAToušEK, J. Approximations and optimal geometric divide-and-conquer. In Proc. 23rd Annu. ACM Sympos. Theory Comput. (1991), pp. 505-511. Also to appear in J. Comput. Syst. Sci.

[16] Mrtchell, J. S., Mount, D. M., AND PapadimIIRIOU, C. H. The discrete geodesic problem. SIAMI J. Comput. 16 (1987), 647-668.

[17] Mount, D. M. Storing the subdivision of a polyhedral surface. Discrete Comput. Geom. 2 (1987), 153-174.

[18] O'Rourke, J. Computational Geometry in C. Cambridge University Press, Cambridge, 1994.

[19] Papadmimtriou, C. H. An algorithm for shortest-path motion in three dimensions. Inform. Process. Lett. 20 (1985), 259-263.

[20] ReF, J. H., AND STORER, J. A. A single-exponential upper bound for finding shortest paths in three dimensions. J. $A C M$ \&1, 5 (1994), 1013-1019.

[21] Sharr, M. On shortest paths amidst convex polyhedra. SIAM J. Comput. 16 (1987), 561-572.

[22] SHARIR, M., AND SchorR, A. On shortest paths in polyhedral spaces. Siam J. Comput. 15 (1986), 193215.

[23] Tan, X.-H., Hirata, T., and Inagaki, Y. Spatial point location and its applications. In Proc. 1st Annes. SIGAL Internat. Sympos. Algorithms (1990), vol. 450 of Lecture Notes in Computer Science, Springer-Verlag, pp. 241-250.

[24] Varadarajan, K., AND Agarwal, P. Approximating shortest paths on a nonconvex polyhedron. In Proc. 38th Annu. IEEE Sympos. Found. Comput. Sci. (1997), pp. 182-191. 